PUPT-1442

hep-th/9401154

\title{
Yangian Symmetry in Conformal Field Theory
}

\author{
Kareljan Schoutens \\ Joseph Henry Laboratories, Princeton University \\ Princeton, NJ 08544, U.S.A.
}

\begin{abstract}
We show that the $S U(N)$, level-1 Wess-Zumino-Witten conformal field theory provides a natural realization of the Yangian $Y\left(s l_{N}\right)$ for $N \geq 3$. We also construct a hamiltonian $\mathrm{H}_{2}$ which commutes with the Yangian generators and study its spectrum. Our results, which generalize work by Haldane et al. [1], provide the field theory extension of the algebraic structure of the $S U(N)$ Haldane-Shastry spin chains with $1 / r^{2}$ exchange.
\end{abstract}

PUPT-1442

hep-th/9401154

January 1994 
Introduction. In the early days of the Wess-Zumino-Witten (WZW) conformal field theory, Polyakov and Wiegmann [2] obtained a number of non-trivial results by applying the Bethe Ansatz method to these theories. Since that time, methods based on conformal invariance have taken over, and have led to a detailed understanding of these conformal field theories. However, for certain applications, especially those where a WZW model arises as an effective description of a many body problem in condensed matter physics, it would be desirable to have an alternative approach to these theories, where by 'alternative' we mean an approach that does not rely directly on the representation theory of the Virasoro algebra or on the associated free field realizations. Recently, Haldane et al. [1] proposed such a new approach to the $S U(2)$, level-1 WZW model. The underlying physical picture is that of an ideal gas of 'spinons', which obey fractional statistics. The method of [1] relies on the Yangian symmetry in the $S U(2)$, level-1 WZW conformal field theory, and we therefore expect that a rather close connection with the Bethe Ansatz work of [2] should exist.

We believe that it would be of definite interest to investigate whether this new approach can be extended to more general conformal field theories. As a first step in this direction, we shall in this Letter present the generalization of the field theory results of [1] to the $S U(N)$, level-1 WZW model for $N \geq 3$.

Yangian symmetry. The Yangian $Y(\mathbf{g})$ associated to a Lie algebra $\mathbf{g}$ is a Hopf algebra that is neither commutative nor cocommutative, and as such it can be viewed as a non-trivial example of a quantum group [3]. Its history goes back to the general formalism of the Quantum Inverse Scattering Method (see [4] for an introduction). Indeed, the object $Y(\mathbf{g})$ is directly related to certain rational solutions of the Quantum Yang Baxter Equation, the simplest of which was first obtained by Yang [5]. In this Letter we focus on the case $\mathbf{g}=s l_{N}$.

We write the lowest generators of $Y\left(s l_{N}\right)$ as $Q_{0}^{a}$ and $Q_{1}^{a}$; higher generators can be obtained by taking successive commutators with the generators $Q_{1}^{a}$. The defining relations of the algebra $Y\left(s l_{N}\right)$ can be written as follows [3]

$$
\begin{aligned}
& {\left[Q_{0}^{a}, Q_{0}^{b}\right]=f^{a b c} Q_{0}^{c},} \\
& {\left[Q_{0}^{a}, Q_{1}^{b}\right]=f^{a b c} Q_{1}^{c},} \\
& {\left[Q_{1}^{a},\left[Q_{1}^{b}, Q_{0}^{c}\right]\right]+(\text { cyclic in } a, b, c)=A^{a b c, \text { def }}\left\{Q_{0}^{d}, Q_{0}^{e}, Q_{0}^{f}\right\},}
\end{aligned}
$$




$$
\begin{aligned}
& {\left[\left[Q_{1}^{a}, Q_{1}^{b}\right],\left[Q_{0}^{c}, Q_{1}^{d}\right]+\left[\left[Q_{1}^{c}, Q_{1}^{d}\right],\left[Q_{0}^{a}, Q_{1}^{b}\right]\right]\right.} \\
& \quad=\left(A^{a b p, q r s} f^{c d p}+A^{c d p, q r s} f^{a b p}\right)\left\{Q_{0}^{q}, Q_{0}^{r}, Q_{1}^{s}\right\}
\end{aligned}
$$

where $A^{a b p, d e f}=\frac{1}{4} f^{a d p} f^{b e q} f^{c f r} f^{p q r}$ and the curly brackets denote a completely symmetrized product. The $S U(N)$ structure constants $f^{a b c}$ have been normalized as

$$
f^{a b c} f^{d b c}=-2 N \delta^{a d} .
$$

The following comultiplications may be used to define the action of the Yangian generators on a tensor product of states

$$
\begin{aligned}
& \Delta_{ \pm}\left(Q_{0}^{a}\right)=Q_{0}^{a} \otimes \mathbf{1}+\mathbf{1} \otimes Q_{0}^{a}, \\
& \Delta_{ \pm}\left(Q_{1}^{a}\right)=Q_{1}^{a} \otimes \mathbf{1}+\mathbf{1} \otimes Q_{1}^{a} \pm \frac{1}{2} f^{a b c} Q_{0}^{b} \otimes Q_{0}^{c} .
\end{aligned}
$$

The 'terrific' (dixit Drinfel'd [3]) right hand sides of the relations (Y3) and (Y4) can be derived from the homomorphism property of these comultiplications. For $\mathbf{g}=s l_{2}$, the cubic relation (Y3) is superfluous and for all other algebras (Y4) follows from (Y2) and (Y3).

Yangian Symmetry in Haldane-Shastry Spin Chains. In 1988, Haldane [6] and Shastry [7] proposed a class of integrable quantum spin chains that are different from those that can be solved by means of the Bethe Ansatz. A characteristic feature is that the spin-spin exchange is not restricted to nearest neighbours. Instead, it has a non-trivial dependence on distance, which, in the simplest case, is of the form $1 / r^{2}$. We shall here recall a few aspects of the simplest of these models. We refer the reader to $[8]$ for a recent account of the state of the art in this field.

The hamiltonian $H_{2}$ of the $S U(N)$ Haldane-Shastry chain with $1 / r^{2}$ exchange acts on a Hilbert space that has $N$ states for each site $i, i=1,2, \ldots, L$. It has the form

$$
H_{2}=\sum_{i \neq j}\left(\frac{z_{i} z_{j}}{z_{i j} z_{j i}}\right)\left(P_{i j}-1\right)
$$

where $P_{i j}$ is a permutation operator that exchanges the states at sites $i$ and $j$, and $z_{i j}=z_{i}-z_{j}$. We choose the complex parameters $\left\{z_{j}\right\}$ as $z_{j}=\omega^{j}$, with $\omega=\exp (2 \pi i / L)$, so that the exchange described by (3) is proportional to the inverse-square of the chord 
distance between the sites. It was found in [1] that the hamiltonian $\mathrm{H}_{2}$ commutes with the following operators

$$
Q_{0}^{a}=\sum_{i} J_{i}^{a}, \quad Q_{1}^{a}=\frac{1}{4} \sum_{i \neq j} \frac{\left(z_{i}+z_{j}\right)}{z_{i j}} f^{a b c} J_{i}^{b} J_{j}^{c},
$$

where the $J_{i}^{a}$ are associated to the action of $S U(N)$ on the $N$ basis states at site $i$. Furthermore, the operators $Q_{0}^{a}, Q_{1}^{a}$ satisfy the defining relations (Y1) - (Y4) of the Yangian $Y\left(s l_{N}\right)$. This remarkable result has been understood to be at the basis of the integrability of these spin chains. Indeed, it appears to be possible [1] to define mutually commuting integrals of motion $H_{n}, n \geq 3$, which commute with the hamiltonian $\mathrm{H}_{2}$ and with the Yangian generators.

Extension to the $S U(2)$, level-1 WZW model. It was pointed out in [1] that the algebraic structure of the $S U(2)$ Haldane-Shastry spin chain has an interesting extension to the case of the $S U(2)$, level-1 WZW conformal field theory. The idea is that in the limit $L \rightarrow \infty$ the sites of the quantum chain become dense on the unit circle and the summation $\sum_{i}$ may be replaced by a contour integral $\oint \frac{d z}{2 \pi i}$. The resulting operators act in the chiral Hilbert space of the $S U(2)$, level-1 WZW conformal field theory. This space is a direct sum of two irreducible highest weight modules of the affine Kac-Moody algebra $A_{1}^{(1)}$, which is generated by currents $J_{m}^{a}$ that satisfy

$$
\left[J_{m}^{a}, J_{n}^{b}\right]=\delta^{a b} m \delta_{m+n}+f^{a b c} J_{m+n}^{c}
$$

In the continuum limit, operator products are assumed to be radially ordered, so that a double sum $\sum_{i, j} f\left(z_{i}, z_{j}\right) J^{a}\left(z_{i}\right) J^{b}\left(z_{j}\right)$ (where $f$ is some function of the $z$ 's) is replaced by the double contour integral $\oint \frac{d z}{2 \pi i} \oint \frac{d w}{2 \pi i} f(z, w) J^{a}(z) J^{b}(w)$, where the contours $\mathcal{O}_{z}$, $\mathcal{O}_{w}$ encircle the origin and $|z|>|w|$. This procedure leads to the following expressions for the Yangian generators $Q_{0}^{a}, Q_{1}^{a}$ and $H_{2}$

$$
\begin{gathered}
Q_{0}^{a}=J_{0}^{a}, \quad Q_{1}^{a}=\frac{1}{2} f^{a b c} \sum_{m>0} J_{-m}^{b} J_{m}^{c}, \\
H_{2}=\sum_{m>0} m J_{-m}^{a} J_{m}^{a} .
\end{gathered}
$$

These operators commute with the zero mode $L_{0}$ of the stress-energy tensor, which has the usual Sugawara form $L_{0}=\frac{1}{2(N+1)} \sum_{m}: J_{-m}^{a} J_{m}^{a}$ : . It is important to notice that $Q_{1}^{a}$ and $H_{2}$ are not zero modes of any conformal fields in the theory. 


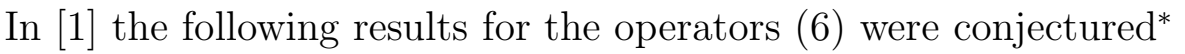

(i) $Q_{0}^{a}$ and $Q_{1}^{a}$ satisfy the generating relations of the Yangian $Y\left(s l_{2}\right)$,

(ii) $Q_{0}^{a}$ and $Q_{1}^{a}$ commutell with $H_{2}$, and

(iii) the spectrum (eigenvalues and eigenspaces) of the operator $H_{2}$ can be obtained by exploiting the representation theory of the algebra $Y\left(s l_{2}\right)$ [9]. The eigenspaces can be characterized by half-infinite sequences of integer rapidities.

The authors of [1] noticed that these field theory results do not directly carry over to algebras different from $S U(2)$. However, in the finite $S U(N)$ Haldane-Shastry spin chains the Yangian $Y\left(s l_{N}\right)$ is realized as an exact symmetry, and this clearly suggests that in $S U(N)$, level-1 conformal field theory a structure based on the Yangian $Y\left(s l_{N}\right)$ should exist. In the remainder of this Letter we shall show that it is indeed possible to modify the expressions (6) for $S U(N)$ in such a way that properties similar to (i)-(iii) [with $Y\left(s l_{2}\right)$ replaced by $Y\left(s l_{N}\right)$ ] can be established. We shall actually prove that the modified operators [given below in (7),(8)] satisfy the properties (i) and (ii), and we shall check that, for the first few levels, the spectrum of $H_{2}$ is consistent with the 'motif' prescription of [1, 10].

Generalization to $S U(N), N \geq 3$. If one takes the expressions (6) for $S U(N)$, $N \geq 3$, one may check that the cubic Serre relation (Y3) of the Yangian and the relation $\left[H_{2}, Q_{1}^{a}\right]=0$ are violated by terms that can be written as zero-modes of conformal fields. This observation suggests that in the case of $S U(N), N \geq 3$, the expressions (6) should be corrected by extra terms that are themselves zero-modes of conformal fields. (There is clearly room for such terms, which could easily appear if the limiting procedure that carries the finite quantum chain over into the field theory would be carried out with greater care.) One more piece of intuition [11] is that the extra term(s) in $H_{2}$ should be such that the degeneracy between conjugate representations

${ }^{*}$ For the algebraic relations ( $i$ ) and (ii) to be valid the action of $Q_{0}^{a}, Q_{1}^{a}$ and $H_{2}$ should be restricted to the irreducible highest weight representations of $A_{1}^{(1)}$ at level one.

${ }^{\dagger}$ It is easily checked that the relation (ii) is also satisfied for level $k=-2$; we have some indications that the irreducible highest weight representations at $k=-2$ (or, for general $S U(N), k=-N$ ) also carry a realization of the Yangian. 
[say, the 10 and the $\overline{10}$ of $S U(3)$ ] is lifted, since the expected eigenvalues of $H_{2}$ are different on these representations.

With this intuition, we arrived at the following Ansatz for the Yangian generators and the hamiltonian $\mathrm{H}_{2}$

$$
\begin{gathered}
Q_{0}^{a}=J_{0}^{a}, \quad Q_{1}^{a}=\frac{1}{2} f^{a b c} \sum_{m>0}\left(J_{-m}^{b} J_{m}^{c}\right)-\frac{N}{2(N+2)} W_{0}^{a}, \\
H_{2}=\sum_{m>0}\left(m J_{-m}^{a} J_{m}^{a}\right)+\frac{N}{(N+1)(N+2)} W_{0} .
\end{gathered}
$$

These expressions contain the zero modes of the following conformal fields (the brackets denote standard normal ordering, see e.g. [12] for conventions)

$$
W^{a}(z)=\frac{1}{2} d^{a b c}\left(J^{b} J^{c}\right)(z), \quad W(z)=\frac{1}{6} d^{a b c}\left(J^{a}\left(J^{b} J^{c}\right)\right)(z)
$$

The 3-index $d$-symbol that occurs in these expressions has been chosen to be completely symmetric and traceless and has been normalized according to

$$
d^{a b c} d^{d b c}=\frac{2\left(N^{2}-4\right)}{N} \delta^{a d} .
$$

Notice that the extra term in $H_{2}$ is proportional to the spin-3 generator of the $\mathcal{W}_{N^{-}}$ algebra of the $S U(N)$, level-1 WZW conformal field theory. It is to be expected that the generators of spin $s \geq 4$ of these algebras will appear as correction terms in the field theory expressions for the higher conserved quantities $H_{n}, n \geq 3$.

We now claim that the operators defined in (7), (8), when acting on irreducible highest weight representations of $A_{N-1}^{(1)}$ at level one, satisfy the properties (i)-(iii) [with $Y\left(s l_{2}\right)$ replaced by $\left.Y\left(s l_{N}\right)\right]$ listed above, and are thus the appropriate generalizations of the $S U(2)$ operators given in (6)). The actual verification of the algebraic properties (i) and (ii) is rather involved, and we shall only briefly explain these computations here. After that we will discuss the spectrum of $\mathrm{H}_{2}$.

Let us first focus on the defining relations of the Yangian $Y\left(s l_{N}\right)$. For $N \geq 3$ the quartic Serre relation (Y4) actually follows from the other relations and the only non-trivial relation to be checked is the cubic relation (Y3). If we write the left hand side of this relation as

$$
f^{b c d}\left[Q_{1}^{a}, Q_{1}^{d}\right]+(\text { cyclic in } a, b, c),
$$


we immediately see that terms in $\left[Q_{1}^{a}, Q_{1}^{d}\right]$ that are of the form $f^{a d e} X^{e}$ do not contribute. We may thus compute modulo such terms. Using only the first terms in $Q_{1}^{a}$ and $Q_{1}^{d}$, this gives the following contribution to $\left[Q_{1}^{a}, Q_{1}^{d}\right]$

$$
\frac{1}{24}\left(f^{a b c} f^{c e g} f^{d e f}-f^{d b c} f^{c e g} f^{a e f}\right)\left\{\left(J^{b}\left(J^{f} J^{g}\right)\right)_{0}-\left(J_{0}^{g} J_{0}^{f} J_{0}^{b}\right)\right\} .
$$

The cross terms involving the first and the second terms in $Q_{1}$ do not contribute; using the second term twice we find a contribution

$$
\begin{aligned}
& \left(\frac{N}{2(N+2)}\right)^{2}\left\{\left(d^{a b c} d^{d e f} f^{c e g}-d^{d b c} d^{a e f} f^{c e g}\right)\left(J^{b}\left(J^{f} J^{g}+J^{g} J^{f}\right)\right)_{0}\right. \\
& \left.+(N+2) d^{a b c} d^{d f c}\left(\partial J^{b} J^{f}-\partial J^{f} J^{b}\right)_{0}\right\}
\end{aligned}
$$

It is easily checked that the term cubic in $J_{0}$ in (11) precisely leads to the desired right hand side of (Y3). We should thus prove that the sum of the remaining terms, when evaluated modulo terms of the form $f^{a d e} X^{e}$, vanishes. In order to proceed we recall that on the Hilbert space of the $S U(N)$, level-1 WZW model the following identity holds

$$
\begin{aligned}
\left(J^{a} J^{b}\right)(z)= & \frac{1}{2} f^{a b c} \partial J^{c}(z)+\frac{1}{N^{2}-1} \delta^{a b}\left(J^{s} J^{s}\right)(z) \\
& +\frac{N}{\left(N^{2}-4\right)} d^{a b c} W^{c}(z)+\frac{N^{2}}{288(N-2)^{2}} T^{a b, c d} V^{c d}(z),
\end{aligned}
$$

where $W^{c}$ is as in (8) and $V^{c d}$ is defined as

$$
V^{c d}(z)=\frac{1}{2} T^{c d, e f}\left(J^{e} J^{f}\right)(z)
$$

This identity implies that the symmetric product of the currents $J^{a}$ and $J^{b}$ decomposes into three terms that transform as a singlet (1), an adjoint representation $\left(N^{2}-1\right)$, and, for $N \geq 4$, a third representation with Dynkin labels $(010 \ldots 010)$ and dimension $\frac{1}{4}(N-3) N^{2}(N+1)$. The tensor $T^{a b, c d}$ that projects on this representation is given by

$$
\begin{aligned}
T^{a b, c d}= & d^{a b c d}+\frac{2 N\left(N^{2}-9\right)}{\left(N^{2}-1\right)\left(N^{2}+1\right)}\left[2 \delta^{a b} \delta^{c d}-\left(N^{2}-1\right)\left(\delta^{a c} \delta^{b d}+\delta^{a d} \delta^{b c}\right)\right] \\
& +\frac{2(N-3)}{N}\left[\frac{2 N}{N^{2}-1} \delta^{a b} \delta^{c d}+N\left(\delta^{a c} \delta^{b d}+\delta^{a d} \delta^{b c}\right)+\left(f^{a c e} f^{e b d}+f^{a d e} f^{e b c}\right)\right],
\end{aligned}
$$


where we defined the completely symmetric, traceless 4-index $d$-symbol as

$$
d^{a b c d}=d^{a b e} d^{e c d}+d^{a c e} d^{e b d}+d^{a d e} d^{e b c}-\frac{4\left(N^{2}-4\right)}{N\left(N^{2}+1\right)}\left(\delta^{a b} \delta^{c d}+\delta^{a c} \delta^{b d}+\delta^{a d} \delta^{b c}\right) .
$$

By using the relation (13) and a variety of contraction identities among the tensors $f^{a b c}, d^{a b c}$ and $d^{a b c d}$ one can work out the remaining terms in (11) and (12). If one then uses that the following dimension-3 fields are both null,

$$
\begin{aligned}
\Phi_{3}^{a d}(z)= & \left(f^{a h p} d^{p b d}-f^{d h p} d^{p b a}\right)\left(J^{b} W^{h}\right)(z) \\
& +(N+2)\left(\partial J^{d} J^{a}-\partial J^{a} J^{d}\right)(z) \\
\Psi_{3}^{a d}(z)= & \frac{N^{2}}{288(N-2)^{2}}\left(f^{a b c} T^{c d, p q}-f^{d b c} T^{c a, p q}\right)\left(J^{b} V^{p q}\right)(z) \\
& -\frac{N(N-3)}{N-2}\left(\partial J^{d} J^{a}-\partial J^{a} J^{d}\right)(z),
\end{aligned}
$$

one finds that indeed the remaining terms in (11), (12) cancel. This then proves the validity of the cubic Serre relation (Y3) and shows that the generators $Q_{0}^{a}, Q_{1}^{a}$ indeed generate the Yangian $Y\left(s l_{N}\right)$.

The fact that the operator $H_{2}$ defined in (7), (8) commutes with the Yangian generators can be established in a similar fashion. With a certain amount of gymnastics, the commutator $\left[Q_{1}^{a}, H_{2}\right]$ can be reduced to the zero mode of the following dimension-4 conformal field

$$
\begin{aligned}
\Xi_{4}^{a}(z)= & \frac{1}{6} f^{a b c} f^{c d e}\left(\partial J^{b}\left(J^{d} J^{e}\right)-J^{b}\left(\partial J^{d} J^{e}\right)\right)(z) \\
& +\frac{N^{2}}{4(N+2)^{2}} d^{a b c}\left[-\frac{2}{3}\left(W^{b} \partial J^{c}+\partial J^{b} W^{c}\right)(z)+\frac{1}{3}\left(\partial W^{b} J^{c}+J^{b} \partial W^{c}\right)(z)\right] \\
& +\frac{1}{24} N(N-2) \partial^{3} J^{a}(z) .
\end{aligned}
$$

A lengthy but straightforward computation shows that this conformal field is actually null, and this then establishes that $H_{2}$ commutes with the generators of the Yangian.

The spectrum of $\mathrm{H}_{2}$. The Hilbert space of the $S U(N)$, level-1 WZW conformal field theory is a direct sum of $N$ irreducible highest weight representations of the affine Kac-Moody algebra $A_{N-1}^{(1)}$. Each of these 'primary sectors' is graded by the eigenvalue of $L_{0}$. The unitarity of these representations guarantees that they are 
completely reducible under the action of the Yangian, and that the operator $H_{2}$ can be diagonalized. The Yangian generators commute with $L_{0}$ so that the $L_{0}$ eigenspaces, which are all finite dimensional, decompose into a finite sum of (finite dimensional) representation of the algebra $Y\left(s l_{N}\right)$. On each of these, $H_{2}$ (and similarly the $H_{n}$, $n \geq 3$ ) takes a constant value.

The authors of [1] proposed a scheme for determining the eigenvalues and eigenspaces of $\mathrm{H}_{2}$. Adapted to the present case, it leads to the following prescription. Each eigenspace is characterized by a set $\left\{m_{1}, m_{2}, \ldots\right\}$ of distinct integer rapidities $m_{i}>0$. These sets can be transformed into sequencses of 0 's and 1's, where a 0 (1) indicates the absence (presence) of the integer that corresponds to the position in the sequence. It is required that (i) at most $(N-1)$ consecutive 1's can occur, and (ii) asymptotically the sequence stabilizes on the pattern 11...11011...11011..., which alternates between single 0's and bunches of $(N-1) 1$ 's. There are $N$ possible 'phases' for the tail of a sequence, and these correspond to the $N$ primary sectors of the $S U(N)$, level-1 WZW model.

The rapidity sequences for states that are descendants w.r.t. the affine algebra $A_{N-1}^{(1)}$ can thus be characterized as finite rearrangements $\left\{m_{i}^{0}\right\} \rightarrow\left\{m_{i}\right\}$ of the corresponding primary state sequence. The eigenvalues of $L_{0}$ and $H_{2}$ are then determined as

$$
\begin{aligned}
& L_{0}=\frac{1}{2(N+1)} C_{2}(R)+\sum_{i}\left[m_{i}^{0}-m_{i}\right], \\
& H_{2}=\frac{N}{6(N+1)(N+2)} C_{3}(R)+\sum_{i}\left[\left(m_{i}^{0}\right)^{2}-\left(m_{i}\right)^{2}\right],
\end{aligned}
$$

where $C_{2}(R)$ and $C_{3}(R)$ are the eigenvalues of the Casimir operators $J_{0}^{a} J_{0}^{a}$ and $d^{a b c} J_{0}^{a} J_{0}^{b} J_{0}^{c}$ in the $S U(N)$ representation $R$ carried by the primary state $\left\{m_{i}^{0}\right\}$. It is expected that the eigenvalues of the $H_{n}, n \geq 3$ will be given by analogous expressions, which, for $n \leq(N-1)$, will involve the eigenvalue of the order- $(n+1)$ Casimir operator of $S U(N)$ in the representation $R$.

The paper [1] also describes a procedure to break up a rapidity sequence into a sequence of 'motifs', which can the be used to find an upper bound on the $S U(N)$ representation content of the corresponding Yangian multiplet.

We have studied the spectrum of the newly constructed operator $H_{2}$ given in (17) and checked that its eigenvalues and eigenspaces are consistent with the above prescription. This once again confirms that the operators (7) are the appropriate field 
theory extensions of the finite chain expressions. As an illustration, we present the results for the first few levels of the three primary sectors for $S U(3)$ in Table 1, and the results for the first four levels of the vacuum representation for $S U(5)$ in Table 2. For general $N \geq 4$, the eigenvalues of $H_{2}$ in the vacuum module are $H_{2}=1$ for $L_{0}=1$, $H_{2}=4,2 N+2$ for $L_{0}=2, H_{2}=5,9,2 N+5,6 N+3$ for $L_{0}=3$, etc. Notice that the $S U(5)$ representation content $(24+75)$, which occurs twice in Table 2 , cannot be viewed as a free product of $S U(5)$ representations (in the product $10 \times \overline{10}$ the singlet drops out). To our knowledge, the systematics of the occurence of such 'incomplete' multiplets, which have been studied in [10], has not been understood completely.

Conclusions. In this Letter we proved that the operators $Q_{0}^{a}$ and $Q_{1}^{a}$ defined in (7), (8) generate the Yangian $Y\left(s l_{N}\right)$, and that they commute with the 'hamiltonian' $\mathrm{H}_{2}$. We also checked that the conjectures of [1] concerning the spectrum of $\mathrm{H}_{2}$ are consistent with an explicit evaluation of $H_{2}$ on a number of low-lying states in the spectrum of the $S U(N)$, level-1 WZW model.

An obvious question is how general the appearance of Yangian symmetry in conformal field theory really is. Already at the level of WZW models the situation is not clear. For instance, if we stay at level $k=1$ but change the Lie algebra to one that is different from $S U(N)$, we find that in general the original Ansatz (6) will not do. On the other hand, modified expressions such as those in (7) cannot be written, simply because algebras different from $S U(N)$ do not have invariant 3 -index $d$-symbols. The situation for higher levels $k \geq 2$ is equally unclear. One may expect that in general rational conformal field theories a structure based on a suitable generalization of Yangian symmetry may be defined. Such a generalized Yangian would then be associated to a general Chiral Algebra, rather than to a Lie algebra. An obvious tool for investigating this issue would be the Goddard-Kent-Olive coset construction. We leave these matters for future investigations 13 .

Acknowledgements. It is a pleasure to thank D. Haldane for drawing my attention to this problem and for illuminating discussions. I also thank E. Frenkel, Z. Ha, V. Korepin and in particular P. Bouwknegt for extensive discussions on this topic. This research was supported by DOE grant DE-AC02-76ER-03072. 


\section{References}

[1] F.D.M. Haldane, Z.N.C. Ha, J.C. Talstra, D. Bernard and V. Pasquier, Phys. Rev. Lett. 69 (1992) 2021.

[2] A.M. Polyakov and P.B. Wiegmann, Phys. Lett. 141B (1984) 223.

[3] V.G. Drinfel'd, Sov. Math. Dokl. 32 (1985) 254; in Proceedings of the International Congress of Mathematicians, Berkeley, California (1986).

[4] V.E. Korepin, G. Izergin and N.M. Bogoliubov, Quantum Inverse Scattering Method, Correlation Functions and Algebraic Bethe Ansatz, Cambridge University Press (1992).

[5] C.N. Yang, Phys. Rev. Lett. 19 (1967) 1312.

[6] F.D.M. Haldane, Phys. Rev. Lett. 60 (1988) 635.

[7] B.S. Shastry, Phys. Rev. Lett 60 (1988) 639.

[8] F.D.M. Haldane, Physics of the Ideal Semion Gas: Spinons and Quantum Symmetries of the Integrable Haldane-Shastry Spin Chain, to appear in the Proc. of the 16th Taniguchi Symposium, edited by A. Okiji and N. Kawakami (Springer, 1994).

[9] V. Chari and A. Pressley, L'Enseignement Math. 36 (1990) 267; J. reine angew. Math. 417 (1991) 87.

[10] Z.N.C. Ha and F.D.M. Haldane, Phys. Rev. B47 (1993) 12459.

[11] F.D.M. Haldane, private communication.

[12] P. Bouwknegt and K. Schoutens, Phys. Rep. 223 (1993) 183.

[13] P. Bouwknegt and K. Schoutens, work in progress. 


\begin{tabular}{||r|r|c|l|l||}
\hline$L_{0}$ & $H_{2}$ & $S U(3)$ irreps & $\left\{m_{i}\right\}$ & 'motifs' \\
\hline 0 & 0 & 1 & $\{1,2,4,5,7,8, \ldots\}$ & $(11)(11) \ldots$ \\
1 & 1 & 8 & $\{2,4,5,7,8, \ldots\}$ & $(01)(11) \ldots$ \\
2 & 4 & 8 & $\{1,4,5,7,8, \ldots\}$ & $(10)(11) \ldots$ \\
2 & 8 & $1+8$ & $\{2,3,5,7,8, \ldots\}$ & ()$(11)(1)(11) \ldots$ \\
3 & 5 & 10 & $\{4,5,7,8, \ldots\}$ & $(00)(11) \ldots$ \\
3 & 11 & $1+8_{2}+\overline{10}$ & $\{1,3,5,7,8, \ldots\}$ & $(1)(1)(1)(11) \ldots$ \\
3 & 21 & $1+8$ & $\{2,3,5,6,8, \ldots\}$ & ()$(11)(11)(1)(11) \ldots$ \\
\hline$\frac{1}{3}$ & $-\frac{1}{9}$ & 3 & $\{2,3,5,6, \ldots\}$ & ()$(11)(1) \ldots$ \\
$\frac{4}{3}$ & $\frac{26}{9}$ & $3+\overline{6}$ & $\{1,3,5,6, \ldots\}$ & $(1)(1)(11) \ldots$ \\
\hline$\frac{1}{3}$ & $\frac{1}{9}$ & $\overline{3}$ & $\{1,3,4,6,7 \ldots\}$ & $(1)(11)(1) \ldots$ \\
$\frac{4}{3}$ & $\frac{10}{9}$ & 6 & $\{3,4,6,7, \ldots\}$ & $(0)(11) \ldots$ \\
$\frac{4}{3}$ & $\frac{46}{9}$ & $\overline{3}$ & $\{1,2,4,6,7 \ldots\}$ & $(11)(1)(11) \ldots$ \\
\hline
\end{tabular}

Table 1: Spectrum of $H_{2}$ on the lowest levels of the irrreducible highest weight representations of $A_{2}^{(1)}$ in the $S U(3)$, level-1 WZW model. The eigenspaces of $H_{2}$ are irreducible representations of the Yangian $Y\left(s l_{3}\right)$. The 'motifs', which have been constructed as in [1], encode information on the $S U(3)$ representation content of the Yangian multiplets, according to $(11)=1,(1)=\overline{3},()=3,(0)=6,(01)=(10)=8,(00)=10$, etc. 


\begin{tabular}{||r|r|c|l|l||}
\hline$L_{0}$ & $H_{2}$ & $S U(5)$ irreps & $\left\{m_{i}\right\}$ & 'motifs' \\
\hline 0 & 0 & 1 & $\{1,2,3,4,6,7,8,9, \ldots\}$ & $(1111)(1111) \ldots$ \\
1 & 1 & 24 & $\{2,3,4,6,7,8,9, \ldots\}$ & $(0111)(1111) \ldots$ \\
2 & 4 & $24+75$ & $\{1,3,4,6,7,8,9, \ldots\}$ & $(1)(11)(1111) \ldots$ \\
2 & 12 & $1+24$ & $\{2,3,4,5,7,8,9, \ldots\}$ & ()$(1111)(111)(1111) \ldots$ \\
3 & 5 & 126 & $\{3,4,6,7,8,9, \ldots\}$ & $(0011)(1111) \ldots$ \\
3 & 9 & $24+75$ & $\{1,2,4,6,7,8,9, \ldots\}$ & $(11)(1)(1111) \ldots$ \\
3 & 15 & $1+24_{2}+75+\overline{126}$ & $\{1,3,4,5,7,8,9, \ldots\}$ & $(1)(111)(111)(1111) \ldots$ \\
3 & 33 & $1+24$ & $\{2,3,4,5,7,8,9,10,12, \ldots\}$ & ()$(1111)(1111)(111) \ldots$ \\
\hline
\end{tabular}

Table 2: Spectrum of $H_{2}$ on the lowest levels of the vacuum representation of $A_{4}^{(1)}$ in the $S U(5)$, level-1 WZW model. The 'motifs' are related to $S U(5)$ representations according to $(1111)=1,(111)=\overline{5},(11)=\overline{10},(1)=10,()=5,(0111)=24$, $(0011)=126$, etc. Note the occurence of the 'incomplete' multiplets $(24+75)$, which arise as $10 \times \overline{10}=1+24+75$, with the singlet dropping out. 